\title{
BIDIRECTIONAL TACHYCARDIA
}

\author{
BY \\ G. C. SEPAHA, S. R. JAIN, AND C. R. BHANDARI \\ From the Seksaria Cardiology Department, M.G.M. Medical College, Indore, India \\ Received January 1, 1961
}

Bidirectional tachycardia is the least common of all arrhythmias and so far only 35 examples (Hellman and Lind, 1956) have been reported and none of them to our knowledge from our country. Schwensen (1922) described the first case which occurred as a result of digitalis intoxication. Palmer and White (1928) reviewed the literature, added cases of their own and discussed the mode of production, suggesting the possibility of a double ventricular circus movement as the most likely mechanism; they named it bidirectional paroxysmal ventricular tachycardia with rhythmic alteration in the direction of the ventricular complexes. Zimdahl et al. (1954) regarded the mechanism as due to the action of two separate foci, one in the A-V nodal tissue above the bifurcation of the bundle and the other in the ventricular muscle. Hellman and Lind (1956) added the last case and suggested that there might be a single focus in the A-V nodal tissue above the bifurcation of the bundle with alternate block in the conduction of both branches; he suggested that it should be called bidirectional tachycardia.

The two important factors present in most of these cases are myocardial damage and digitalis therapy. Among the 35 cases reported so far, 28 were receiving digitalis at the time the arrhythmia occurred. Although some patients received toxic doses of digitalis, in the majority of them the dosage was in the average therapeutic range. Digitalis has failed to produce these arrhythmias in the healthy heart and previous myocardial damage is an important factor in precipitating them (Kobacher and Scherf, 1929). The mortality in the previously reported cases indicates a grave prognosis. Of the 35 cases 26 died soon after the arrhythmia was discovered while 5 others died within 2 to 27 months.

The response to potassium salts in various arrhythmias produced by digitalis is well known. Sampson et al. (1943) reported that ectopic beats caused by digitalis could be abolished in every instance by administration of potassium. Enselberg et al. (1950) got similarly striking results by using potassium to abolish multiple ventricular extrasystoles and also bidirectional ventricular tachycardia associated with digitalis therapy.

Experimentally it has been demonstrated that magnesium salts in large doses depress conduction and depress or abolish abnormal impulse formation. These effects have been confirmed clinically in damaged hearts, whereas no important effects were noted in normal hearts. It has also been noted that magnesium is more likely to abolish ectopic beats caused by digitalis than those caused otherwise (Hellman and Lind, 1956).

We now report two patients suffering from bidirectional tachycardia. It was thought it would be of interest to record them in view of the rarity of the condition and the controversial nature of the arrhythmia and its production.

\section{CASE RePorts}

Case 1. A man, aged 50, was admitted to hospital on July 14, 1958 complaining of dyspnoa on exertion for two years. For five years there had been winter coughs. For one month he had been troubled with orthopnœa and œdema had appeared in the feet ten days before admission. 
Examination. The patient was well-built, orthopnœic, and cyanosed. There was generalized œdema, mostly in the feet, while the neck veins were distended. Slight clubbing of the fingers was present. The pulse rate was 140 a minute, and the rhythm was regular. There was no fever but the respiration rate was 40 a minute. The blood pressure measured $100 / 80 \mathrm{~mm}$. $\mathrm{Hg}$.

The apex beat was not palpable. Cardiac dullness was lessened by emphysema. While the heart sounds were generally feeble the pulmonary second sound was relatively accentuated. In the lungs râles and rhonchi were heard at all areas but specially at the lung bases. The abdomen was distended and there was œdema of the abdominal wall with some free fluid in the peritoneal cavity. The liver was enlarged four fingers' breadth below the costal margin and tender. The spleen was not palpable.

Investigation of the urine and blood revealed no material deviation from the normal.

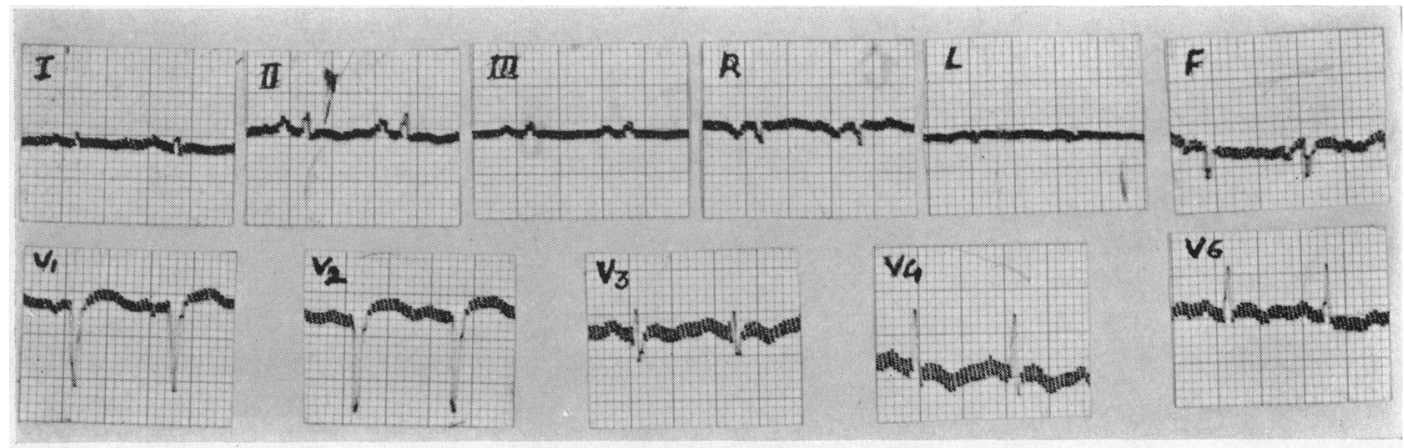

Fig. 1.-(Case 1). E.C.G. on 14/7/58. Sinus rhythm 130/min. Low voltage curve; prominent $P$ wave in lead II; inverted T in leads I, II, and V3 to V6, and flat T in leads III, AVL, and AVF.

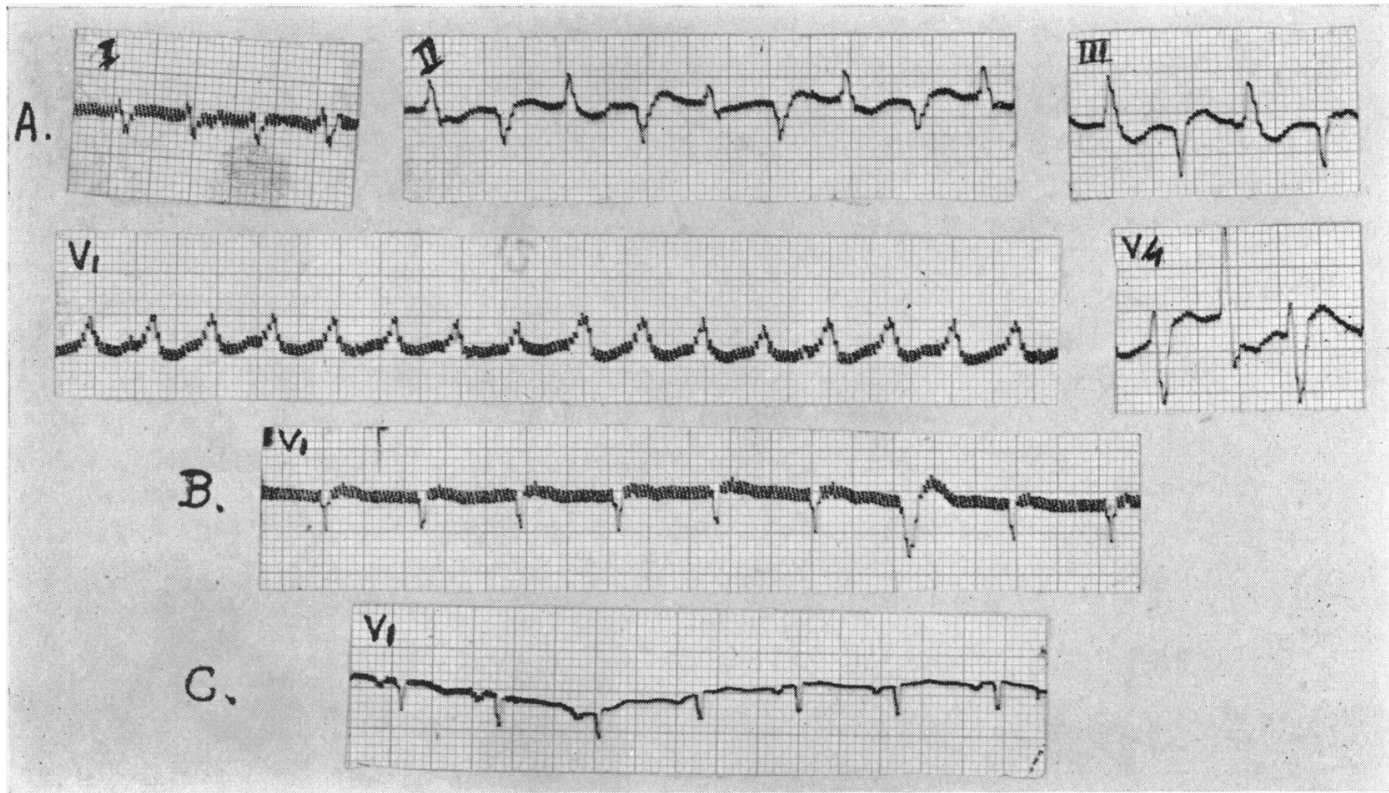

Fig. 2.-(Case 1). (A) E.C.G. 18/7/58. Rate $180 / \mathrm{min}$. Supraventricular tachycardia with partial right bundle-branch block. No definite $P$ waves. In leads II, III, and V4 alternate ventricular complexes are in opposite directions. (B) V1 a few minutes later shows nodal rhythm with slower rate and normal conduction. The retrograde $P$ waves can be made out. (C) V1 still a few minutes later shows sinus rhythm with same rate. 
An electrocardiogram was taken on the day of admission showing sinus rhythm (Fig. 1). On 14/7/58 penicillin, aminophylline, and digoxin $0.25 \mathrm{mgm}$. thrice daily were given. Oxygen and mercurial diuretics were also required. With four days of treatment the patient showed slight clinical improvement, but the pulse rate increased. A further cardiogram showing bidirectional tachycardia (Fig. 2A) was taken on 18/7/58 and treatment by digitalis was stopped. Lead V1 showed supra-nodal tachycardia with partial right bundlebranch block. This was succeeded within a few minutes by nodal rhythm, and a few minutes later, sinus rhythm was recorded (Fig. 2B and C). Later records of this patient taken on 18/7/58 are shown in Fig. 3 and Fig. 4. He died on the following day, no necropsy being permitted.

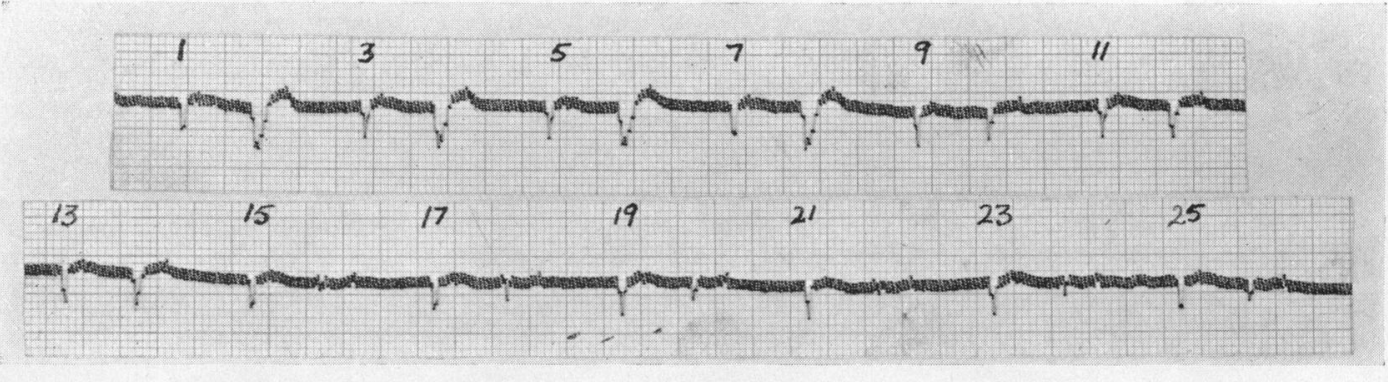

FIG. 3.-(Case 1). E.C.G. 18/7/58. Lead V1 showing nodal rhythm with alternate extrasystoles. First, third and subsequent alternate beats are nodal with retrograde $P$ waves, while second, fourth, etc.are extrasystoles, probably nodal with aberrant conduction because $10 \mathrm{th}, 12 \mathrm{th}$, etc. are also similar nodal extrasystoles but with normal conduction and delayed retrograde conduction to the auricles. The 16th, 18th, etc. and later alternate beats are also the same with a different QRS complex suggesting aberrant conduction.

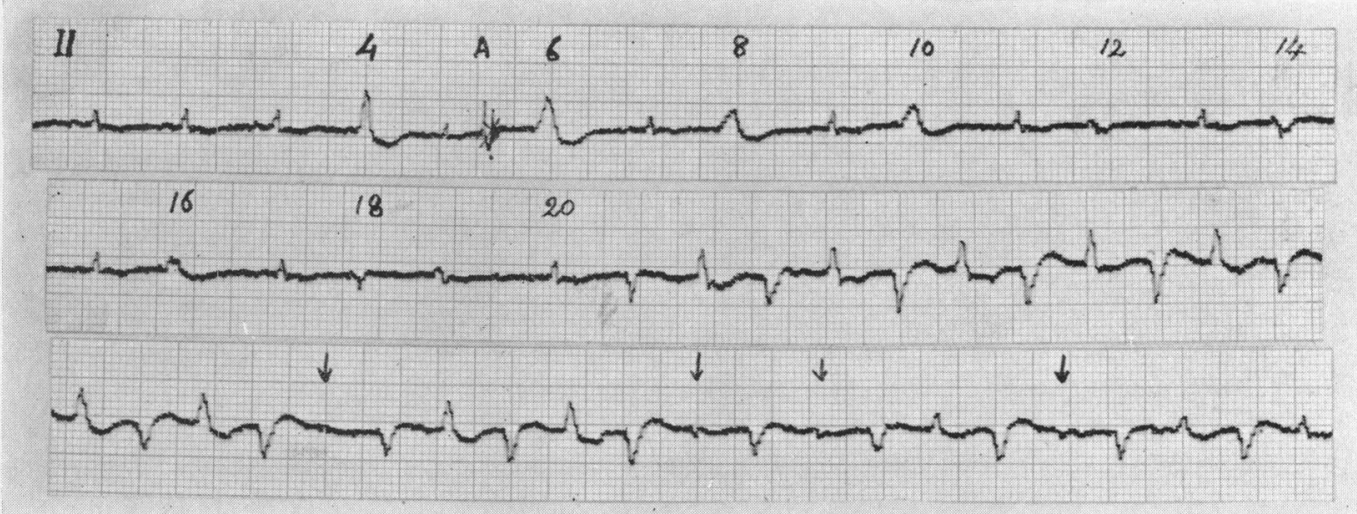

FIG. 4.-(Case 1). E.C.G. 18/7/58. Continuous lead II. The 4th beat comes at regular time but shows aberrant conduction. The 7 th, 9 th, and alternate later beats are sinus beats while 6 th, 8 th, etc., are possibly nodal extrasystoles with aberrant conduction as in Fig. 3. The 20th beat is a sinus beat and then the bidirectional tachycardia sets in. The rhythm is regular and the distance between any two beats is 0.34 sec. The arrow marked beats are blocked beats. $\mathrm{A}=$ Artefact.

Case 2. A woman of 60 was admitted to hospital on March 26, 1959 complaining of paroxysmal dyspnœa over the past 10 years. The attacks used to occur at any time of the day, with no relation to exertion and used to be relieved by symptomatic treatment. For four months the attacks of dyspnœea had been more frequent. She also began to have exertional dyspnœa and cough with slight mucoid expectoration. These symptoms continued with increasing severity until 15 days before admission when she noticed odema of the ankles. Orthopnœa and occasionally slight hæmoptysis followed. There was no significant family or past personal history. 


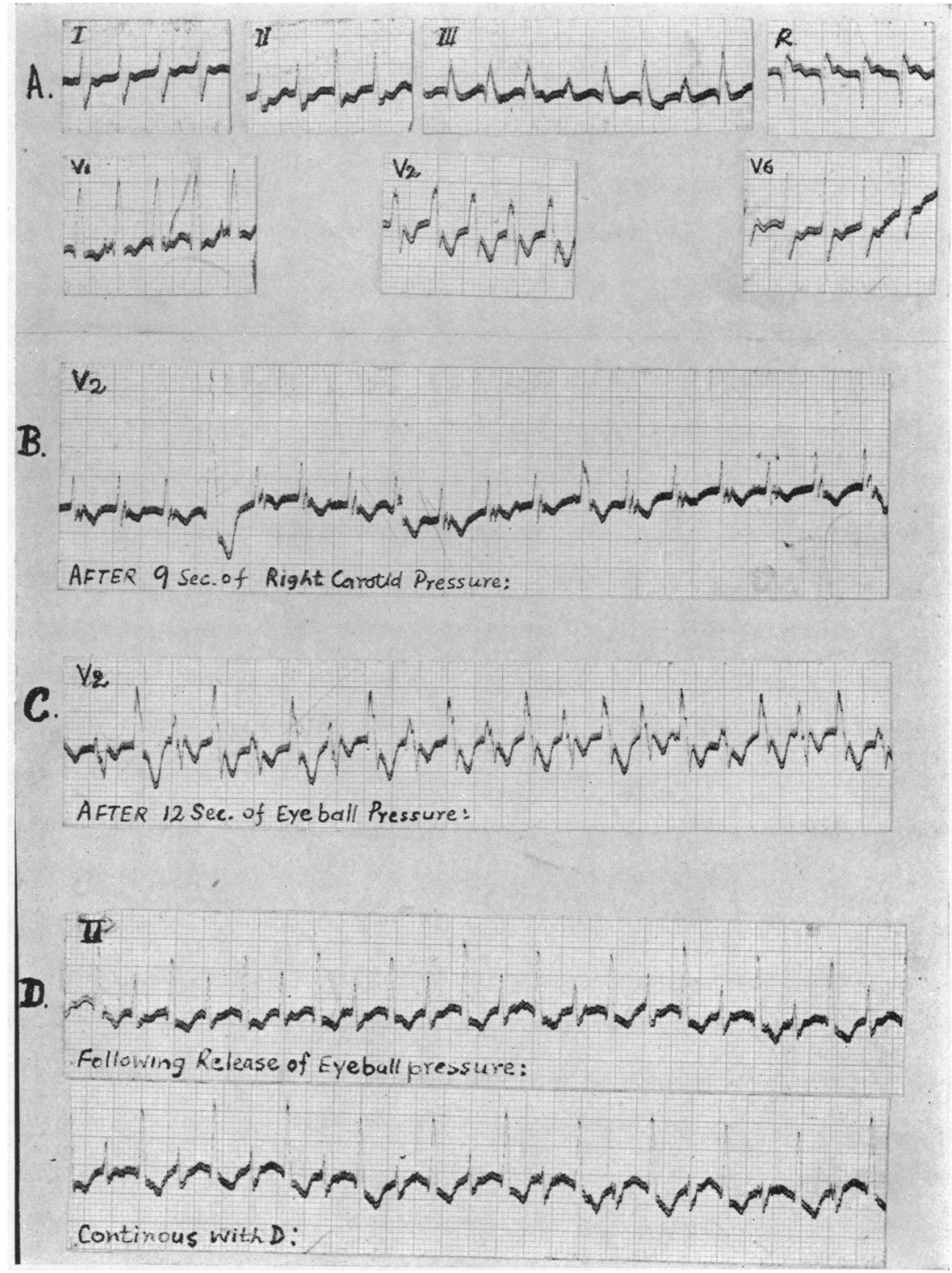

Fig. 5.-(Case 2). E.C.G. 30/3/59 at 1 p.m. (A) Supraventricular tachycardia with right bundle-branch block. Rate $200 / \mathrm{min}$. In lead V1 some P waves can be seen suggesting auricular activity. (B) Lead V2 after right carotid pressure shows slowing of rate to about $160 / \mathrm{min}$. and change in the configuration of ventricular complexes with occasional ventricular extrasystole. (C) Lead V2 after eyeball pressure shows change in configuration of alternate beats suggesting bidirectional tachycardia. Both the types of beats are equidistant. (D) Lead II: alternating beats are gradually changing from unidirectional tachycardia to bidirectional.

Examination. The patient was an orthopnœic and cyanosed woman of average build. Cervical venous congestion, œdema of the legs, signs of pulmonary œdema and hepatic enlargement denoted congestive heart failure.

The rhythm was regular at 140 a minute. The arterial pressure was 130/90. Emphysema precluded localization of the apex beat but epigastric pulsation pointed to right ventricular hypertrophy. The heart 


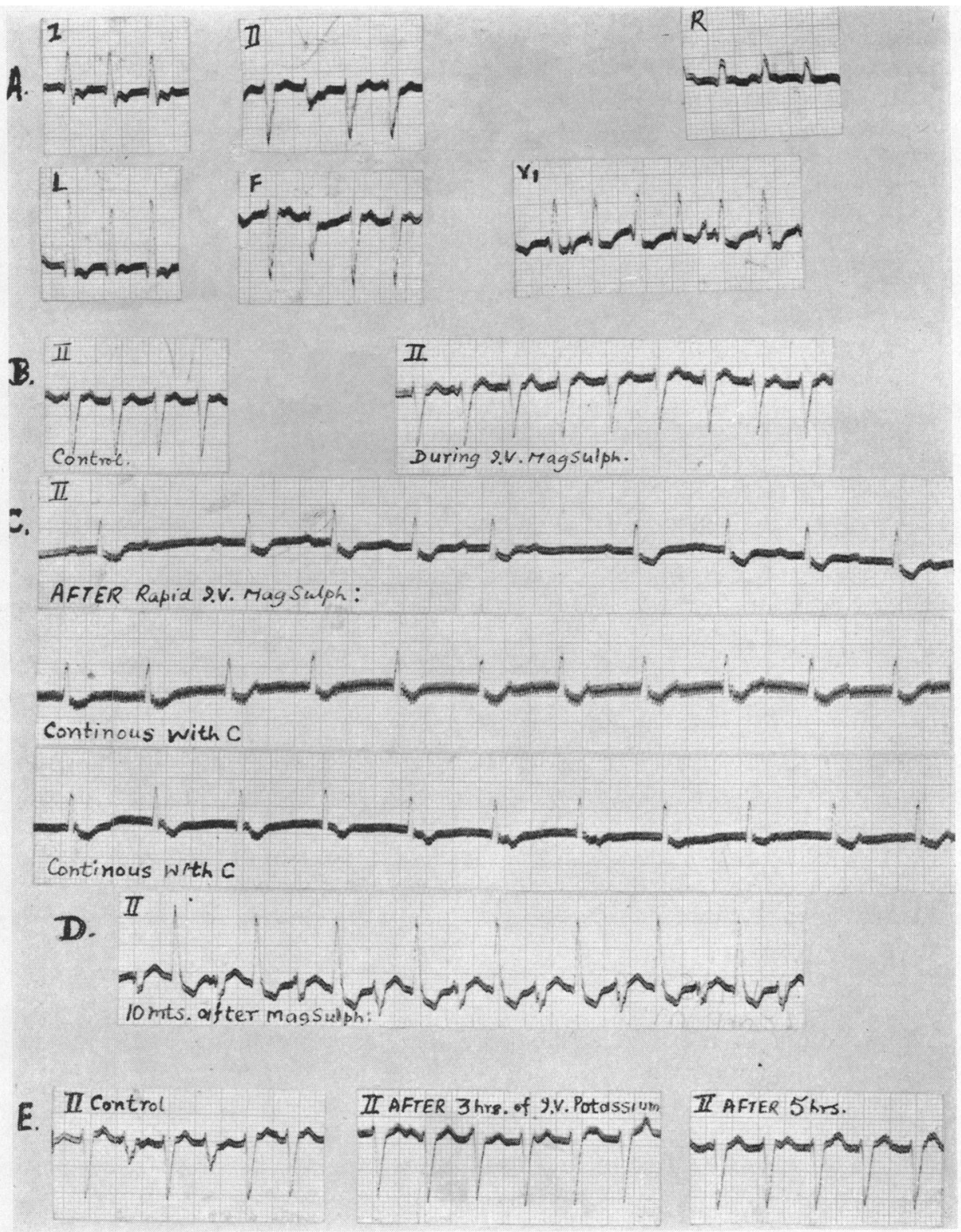

FIG. 6.-(Case 2). E.C.G. $30 / 3 / 59$ at 6 p.m. (A) The tachycardia is chiefly unidirectional of slower rate with few beats showing aberrant conduction. Right bundle-branch block pattern maintained in lead V1. (B) Lead II control and after slow I.V. injection of magnesium sulphate shows slight slowing of rate. (C) Lead II after rapid I.V. injection shows stoppage of tachycardia and A-V dissociation. (D) After 10 minutes reversion to bidirectional tachycardia. (E) 3 and 5 hours after I.V. injection of potassium chloride the cardiogram shows no appreciable change except slight slowing of rate.

sounds were faint except for accentuation of the pulmonary second sound. No murmurs were heard, nor any other added sounds. In the lungs there were râles and rhonchi over both midzones and bases, specially over the left midzone. The enlarged liver was tender; the spleen was not palpable.

Investigation. The urine contained a trace of albumin. The blood count was normal and the Kahn test was negative. X-rays of the chest showed hazy opacity in left middle zone. The electrocardiogram 
showed sinus rhythm at 140 a minute, flat $\mathrm{T}$ waves in lead I and inverted $\mathrm{T}$ waves in lead V4, V5, and V6, suggesting ischæmic heart disease. No digitalis had been given.

Treatment. The patient was given digoxin $0.5 \mathrm{mgm}$. thrice daily, aminophyllin $0.1 \mathrm{~g}$. thrice daily and intramuscularinjections of penicillin. She received only one injection of neptal on the day following admission. The same treatment was continued for four days thereafter. At $1 \mathrm{p} . \mathrm{m}$. on March 30 her pulse rate suddenly rose to 200 a minute. The cardiogram (Fig. 5) was repeated. Digoxin was stopped and administration of potassium chloride $1 \mathrm{~g}$. thrice daily started. The tachycardia persisted, and at 6 p.m. an intravenous injection of magnesium sulphate 12.5 c.c. of 20 per cent solution was given. There was a temporary A-V dissociation rhythm (Fig. 6C) which after about 10 minutes disappeared; the bidirectional tachycardia then restarted (Fig. 6D). At 9 p.m. an injection of glucose, 20 oz. of a 5 per cent solution was given in conjunction with an intravenous drip of $1.5 \mathrm{~g}$. of potassium chloride at the rate of about 25 drops per minute. There was no change in the arrhythmia (Fig. 6E). Two days later (April 1) the cardiogram showed sinus rhythm at the rate of 90 a minute. On the next day at 2 p.m. the tracing showed sinus rhythm at the rate of 100 a minute with right bundle-branch block and multiple ventricular extrasystoles. At 3.30 p.m. the patient died suddenly.

Necropsy Findings. The heart weighed $475 \mathrm{~g}$.; both chambers were hypertrophied and dilated. The mitral valve showed old healed rheumatic vegetations but no stenosis. In the aorta and main coronary arteries there was evidence of early atherosclerosis. Chronic venous congestion was found in the lungs with an infarction in the postero-lateral segment of the left upper lobe. The kidneys showed venous congestion and cloudy swelling, the spleen congestion and infarction, and the liver chronic venous congestion. The histopathological study showed œdema and cellular infiltration in the S-A node and auricular muscle while the A-V node and bundle of His showed minimal changes. The ventricular musculature did not show any abnormality in the form of increased fibrous or inflammatory cells.

\section{DISCUSSION}

Bidirectional tachycardia is a rare but dangerous arrhythmia. Both of the patients presented here died within 50 hours of the onset of the tachycardia. The ætiology in each instance was not different from the cases which have been reported earlier. Case 1 was suffering from chronic bronchitis, emphysema, chronic pulmonary heart disease, ischæmic heart disease and congestive failure and was digitalized by digoxin $0.25 \mathrm{mgm}$. thrice daily for four days. Case 2 had bronchial asthma, emphysema, chronic pulmonary heart disease, ischæmic heart disease, pulmonary infarction, and congestive failure; she was digitalized by digoxin $0.5 \mathrm{mgm}$. thrice daily for four days. Thus it could be said that anoxia, ischæmia of the myocardium and digitalis were the contributing factors in precipitating the tachycardia.

The mechanism of production has been a controversial point in the past. Until 1956 it was supposed to be due to two different foci; both might be in the ventricles or one might be in the ventricle and the other in the A-V nodal tissue; functioning alternately they could produce bidirectional tachycardia (Braun and Wosika, 1945; Palmer and White, 1928). These views were challenged following the observed effects of carotid sinus pressure on the arrhythmia.

In Case 1 and Case 2 the distances between the upward and downward complexes were 0.34 sec. and $0.26 \mathrm{sec}$. respectively and the rhythm in both was absolutely regular, as it was also in the case of Hellman and Zimdahl et al. (1954). In the case described by Hellman and Lind (1956) the distance between upward and downward complexes was $0.36 \mathrm{sec}$., while the distance between downward and subsequent upward complexes was $0.4 \mathrm{sec}$.

Two types of bidirectional tachycardia could exist. In type I the distance between the upward and downward beats and subsequent upward beats is not the same while in the type II the distance is the same; nevertheless, in both of them the distance between the two upward complexes is the same as between two downward complexes. It is possible that in bidirectional tachycardia type I there may be two foci working in the A-V nodal tissue. In type II bidirectional tachycardia there is one focus situated in the A-V nodal tissue, initiating the rhythm, and the impulse passes alternately through each bundle and produces bidirectional tachycardia as in both of our cases.

The tachycardia in Case 1 did not respond to carotid or eyeball pressure though it was spontaneously changing, but before the onset of tachycardia this patient showed nodal rhythm with alternate nodal extrasystoles, some of the later beats showing aberrant conduction (Fig. 3). The 
carotid and eyeball pressure in Case 2 made a change in the configuration of the complexes, possibly due to change in conduction, but what was more striking was the slowing of rate (Fig. 5B) thus showing that the tachycardia was not ventricular in origin. Lead II in Fig. 5D shows unidirectional tachycardia with two types of beats alternately coming regularly. The smaller amplitude beats resemble the beats of tachycardia with right bundle-branch block (Fig. 5A); the other group of alternate beats having bigger amplitude could be partial left bundle-branch block beats. The $R$ wave of the smaller beats is gradually becoming less and less while the $S$ wave gradually becomes more and more till $\mathrm{R}$ completely disappears and only the wide $\mathrm{S}$ remains, possibly due to changing from partial right bundle-branch block to complete RBBB, producing a bidirectional tachycardia.

The cardiogram in Fig. 4A taken a few hours later shows the persistence of tachycardia at a slower rate. This was possibly due to potassium chloride $2 \mathrm{~g}$. which the patient received by mouth during this time.

In Case 2 a slow I.V. injection of 20 per cent magnesium sulphate ( $7 \cdot 5$ c.c. in about 4 minutes) did not make any appreciable change (Fig. 6B) but rapid injection of 5 c.c. did definitely disturb the rhythm, causing a slow dissociation (Fig. 6C). This dissociated rhythm was of short duration and within ten minutes again changed to bidirectional tachycardia (Fig. 6D), a sequence also reported by Hellman and Lind (1956). From the time the tachycardia set in digitalis was stopped and the patient was put on potassium chloride $1 \mathrm{~g}$. thrice daily by mouth; she was also given an I.V. drip of potassium chloride ( $1.5 \mathrm{~g}$. in 500 c.c. of 5 per cent glucose). This made no appreciable change except slight slowing of rate during the time of drip but about 24 hours after the drip was discontinued sinus rhythm set in. Case 1 did not receive magnesium sulphate intravenously but received potassium chloride $1 \mathrm{~g}$. thrice daily by mouth; this brought no response and the patient died within 30 hours of the onset of tachycardia. Potassium and magnesium are the drugs of choice in disorders of this kind but to abort the attack the injection should be used more rapidly than usual. Unfortunately, however, the effect may be evanescent. From histological studies it appears that bidirectional tachycardia could exist without there being gross changes in the ventricular musculature.

It appears that bidirectional tachycardia is not of ventricular but of supra-ventricular nodal origin. It is possible that two foci in the A-V nodal tissue may be responsible in some cases, while one focus with conduction through alternate bundles is the mechanism in other cases. Pulmonary infarction should be considered as a possible cause of bidirectional tachycardia, specially when there is changing bundle-branch block with spontaneous remissions, as in Case 2.

\section{SUMMARY AND CONCLUSIONS}

Two cases of bidirectional tachycardia are reported. The upward and downward complexes were regular in both of our cases, the distance between them being $0.34 \mathrm{sec}$. in one and $0.26 \mathrm{sec}$. in the other. Pressure over the carotid slowed the rate in the second of these two patients; in the first the paroxysmal tachycardia was preceded by nodal rhythm and nodal extrasystoles with conduction defect.

Conversion of unidirectional to bidirectional tachycardia by gradual change from incomplete bundle-branch block in alternate beats, the presence of all types of conduction disturbance, e.g. nodal rhythm, nodal tachycardia with right or with left bundle-branch block, nodal extrasystoles with conduction through right or left bundle and blocked nodal extrasystoles are all in support of this tachycardia being nodal in origin.

Effect of potassium and magnesium on the tachycardia has been described.

The necropsy findings of one case with the results of histopathological study of the heart has been described.

The possible mechanisms of production of this type of tachycardia have been discussed.

Our grateful acknowledgments are due to Dr. S. K. Mukerji, Emeritus Professor of Medicine, for his valuable criticisms and comments, and to Dr. C. M. Rangam, Professor of Pathology, for the necropsy findings. 


\section{REFERENCES}

Braun, L., and Wosika, P. H. (1954). Amer. Heart J., 29, 261.

Enselberg, C. D., Simmons, H. G., and Mintz, A. A. (1950). Amer. Heart J., 39, 713.

Felbergaum, D. (1923). Amer. J. med. Sci., 166, 211.

Hellman, E., and Lind, A. (1956). Amer. Heart J., 51, 140.

Kobacher, G. L., and Scherf, D. Quoted by Hellman and Lind.

Palmer, R., and White, P. D. (1928). Amer. Heart J., 3, 454.

Sampson, J. J., Alberton, E. C., and Kondo, B. (1943). Amer. Heart J., 26, 164.

Schwensen, C. (1922). Heart, 9, 199.

Zimdahl, W. T., and Kramer, L. I. (1947). Amer. Heart J., 33, 218.

- , and Townsend, C. E. (1954). Amer. Heart J., 47, 304. 